\title{
The impacts of allopolyploidization on Methyl-CpG-Binding Domain (MBD) gene family in Brassica napus
}

Yafang Xiao ${ }^{1}$, Mengdi $\mathrm{Li}^{1,2}$ and Jianbo Wang ${ }^{1 *}$

\begin{abstract}
Background: Polyploidization promotes species formation and is widespread in angiosperms. Genome changes dramatically bring opportunities and challenges to plants after polyploidy. Methyl-CpG-Binding Domain (MBD) proteins can recognize and bind to methylation sites and they play an important role in the physiological process related to methylation in animals and plants. However, research on the influence of the allopolyploidization process on the MBD gene family is still lacking, so it is necessary to conduct a comprehensive analysis.

Results: In this study, twenty-two, ten and eleven MBD genes were identified in the genome of allotetraploid $B$. napus and its diploid ancestors, B. rapa and B. oleracea, respectively. Based on the clades of the MBD gene in Arabidopsis, rice and maize, we divided the new phylogenetic tree into 8 clades. Among them, the true MBD genes in Brassica existed in only 5 clades. Clade IV and Clade VI were unique in term of MBD genes in dicotyledons. Ka/Ks calculations showed that $M B D$ genes underwent purifying selection in Brassica and may retain genes through sequence or functional differentiation early in evolution. In the process of allopolyploidization, the number of MBD gene introns increased, and the protein motifs changed. The MBD proteins had their own special motifs in each clade, and the MBD domains were only conserved in their clades. At the same time, the MBD genes were expressed in flower, leaf, silique, and stem tissues, and the expression levels of the different genes were significantly different, while the tissue specificity was not obvious. The allopolyploidization process may increase the number of cis-acting elements and activate the transposable elements. During allopolyploidization, the expression pattern of the MBD gene changes, which may be regulated by cis-acting elements and transposable elements. The number imbalance of cis-acting elements and transposable elements in $A_{n}$ and $C_{n}$ subgenomes may also lead to biased $A_{n}$ subgenome expression of the $M B D$ gene in B. napus.
\end{abstract}

Conclusions: In this study, by evaluating the number, structure, phylogeny and expression of the MBD gene in $B$. napus and its diploid ancestors, we increased the understanding of MBD genes in allopolyploids and provided a reference for future analysis of allopolyploidization.

Keywords: MBD gene family, Allopolyploidization, Brassica napus, Expression pattern, Gene structure

*Correspondence: jbwang@whu.edu.cn

1 State Key Laboratory of Hybrid Rice, College of Life Sciences, Wuhan University, Wuhan 430072, China

Full list of author information is available at the end of the article

\section{Background}

Frequent and recurrent polyploidization events promote speciation and exist widely in the evolutionary history of plants [1-4]. Polyploidy provides the potential for genetic diversity while increasing the cost of plant genome replication and epigenetic instability [5]. Genomic changes caused by polyploidization are mostly nonadditive, such 
as genome reorganization or functional modifications [6]. Although gene expansion occurs from time to time during the process of polyploidization [7], the polyploid genome is not simply a direct doubling of diploid genomes. Extreme changes in the genome during the early stage of the formation of neopolyploids may lead to events such as DNA sequences and gene loss [8]. Gene expansion caused by polyploidization may eventually return to one gene copy through separation and loss, or it is possible that multiple copies of one gene develop different functional trends [2]. In addition, allopolyploid plants rely on the richness of their genomes, which has a significant impact on trait diversification, crop domestication, and environmental adaptation $[4,9,10]$.

As an epigenetic modification, DNA methylation plays a significant role in gene expression regulation and genome stability [11]. The most well-known modifications of DNA-recognition domains are the SET and RING finger-associated domain (SRA) and the MethylCpG-Binding Domain (MBD) [12]. MBD proteins usually recognize symmetrically CG-methylated oligonucleotides, but studies have shown that they can also bind to $\mathrm{mCA}$ sites [13]. The importance of MBD proteins has led to heated research on their structure and function. It was found that MBD5 and MBD6 in Arabidopsis thaliana can be recruited into chromatin through the recognition of CG methylation sites, repression of gene subsets and transposons, and the maintenance of unchanged DNA methylation levels [13]. MBD7 protects genes from expression repression and DNA hypermethylation by binding to the highly methylated CG region and recruiting histone acetyltransferases [14]. MBD9 strongly interacts with Imitation SWItch (ISWI) chromatin remodeling complexes and has been shown to be involved in DNA demethylation [15-17]. In Arabidopsis mutants with $M B D$ gene knockdown, nucleolar dominance was disturbed, and their growth and development were abnormal [18]. In mammals, MBD proteins are involved in tumorigenesis, schizophrenia, pulmonary fibrosis and many other diseases $[19,20]$.

The Brassica genus has excellent crop characteristics and a profound cultivation history. They experienced an additional whole-genome triplication (WGT) event after differentiation from Arabidopsis [21, 22]. Following WGT, gene loss events resulted in three subgenomes of the least fractionated blocks (LF), the medium fractionated blocks (MF1) and the most fractionated blocks (MF2) in B. rapa [23]. Brassicaceae share the same ancestral karyotypes, basic genomic blocks, and significant synteny between genomes [24]. Brassica napus (AACC, $2 \mathrm{n}=4 \mathrm{x}=38$ ), an allotetraploid plant, is formed by interspecific crosses between two diploid ancestors, Brassica rapa (AA, $2 \mathrm{n}=2 \mathrm{x}=20$ ) and Brassica oleracea
(CC, $2 n=2 x=18)$, followed by whole-genome duplication (WGD) events and it is an excellent material for studying plant polyploidy [25]. To date, $M B D$ genes have been identified and studied in Arabidopsis, rice, maize and other plants [26], but the influence of the process of allopolyploidy on this gene family is still unknown in the Brassica genus. In this study, based on the Arabidopsis MBD protein sequence, MBD proteins and genes were identified in B. rapa, B. oleracea, and B. napus. We explored the evolutionary experience of the $M B D$ gene family and enhanced the understanding of the impact of allopolyploidization by analyzing the chromosome location, phylogenetic tree, syntenic genes, gene structure, evolutionary pressure, cis-acting elements, and gene expression patterns of $M B D$ genes.

\section{Results}

\section{Identification of the $M B D$ genes}

To accurately identify $M B D$ gene family members, we considered that only those with complete MBD domains were MBD proteins. Thirteen putative MBD proteins in $A$. thaliana [26] were used as query sequences, and BLASTp was used to search for B. rapa, B. oleracea, and B. napus in the BRAD database (http://brassicadb.cn/\#/). All of the identified candidate protein sequences were confirmed by the CDD, Pfam and InterPro databases, and the proteins with complete MBD domains that could be queried in all three databases were retained. Finally, ten genes encoding the MBD proteins were identified in $B$. rapa, eleven in $B$. oleracea and twenty-two in $B$. napus. Based on their homology with Arabidopsis $M B D$ genes, they were named $B r M B D 2$ to $B r M B D 11 b$ in $B$. rapa, $B o M B D 2$ to BoMBD11 in B. oleracea, and BnCMBD2a to $B n A M B D 11 d$ in $B$. napus. When homologous to the same Arabidopsis gene, the last letter of the name, from "a" to "g", indicates a gradual decline in homology, and in $B$. napus, the A and C letters after "Bn" indicate the $B$. napus $A_{n}$ subgenome and $C_{n}$ subgenome. The total number of $M B D$ gene family members in diploid ancestors was less than that in tetraploid $B$. napus, indicating expansion events of the $M B D$ gene during the formation and evolution of B. napus.

\section{Chromosomal location and duplication pattern analysis of $M B D$ genes}

The locations of the $M B D$ genes of $B$. napus and its diploid ancestors on the chromosomes were displayed using MapInspect software. In addition to $B n C M B D 4 c, B n C$ $M B D 6 a$ and BnAMBD10e in B. napus and BoMBD9 in $B$. oleracea, which were located on the unassembled scaffold, the remaining $39 M B D$ genes were located on the chromosomes of each species, respectively (Fig. 1). Ten B. rapa $M B D$ genes were located on 8 chromosomes 


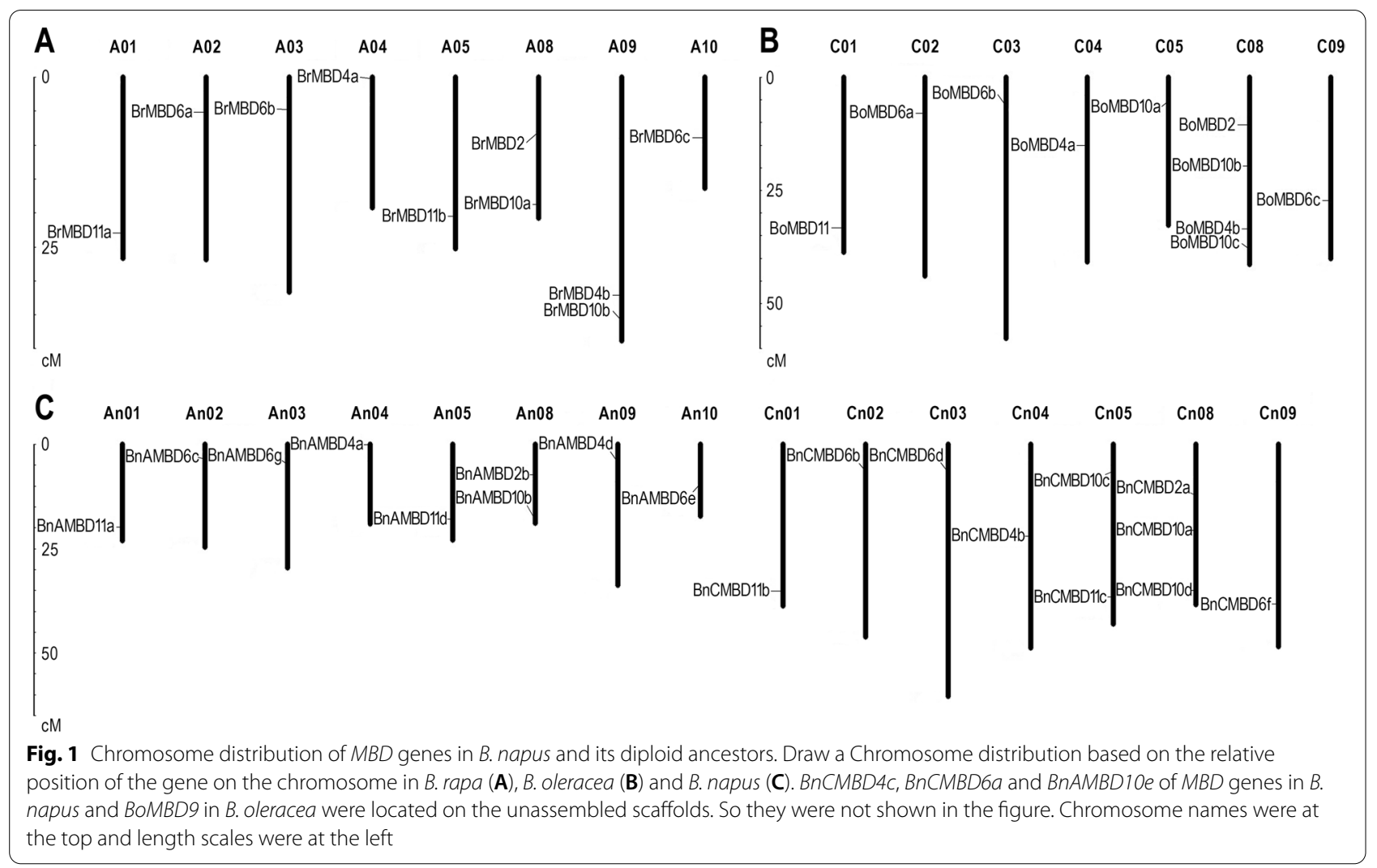

(Fig. 1A), ten B. oleracea $M B D$ genes were located on 7 chromosomes (Fig. 1B), and nineteen B. napus $M B D$ genes were located on 15 chromosomes (Fig. 1C). Comparing the distribution of $M B D$ genes on the B. napus $\mathrm{A}_{\mathrm{n}}$ subgenome with the $B$. rapa A genome, the gene homology and their relative position on the chromosome were the same, except that $A_{n} 09$ may have gene loss or incomplete chromosome assembly. Similarly, comparing the gene distribution of the $B$. napus $C_{n}$ subgenome with the $B$. oleracea $C$ genome, one gene was added to $C_{n} 05$, a gene on $\mathrm{C}_{\mathrm{n}} 08$ was not located, and the remaining genes did not change their positions on the chromosome during the formation of $B$. napus. Relatively speaking, the $A_{n}$ subgenome was more stable than the $C_{n}$ subgenome, but overall, the $M B D$ gene distribution on the chromosome did not change significantly during the process of hybridization and polyploidization.

More than one WGD event occurred during the formation of the ancestral polyploidy Brassica genus, and the formation of B. rapa and B. oleracea genomes was inseparable from whole genome triplication and extensive diploidization [27]. During evolution, the maintenance of gene family members often depends on different duplication modes [28]. We counted the members of the $M B D$ genes according to the five duplication modes, i.e., WGD, TD (tandem duplication), PD (proximal duplication), TRD (transposed duplication), and DSD (dispersed duplication) and found that WGD, TRD and DSD, especially WGD and TRD, played a major role in the increase in $M B D$ gene numbers in B. rapa, B. oleracea and B. napus (Additional file 1: Table S1). WGD events played an important role in B. rapa (8/10) and B. oleracea (9/11), but the importance of its tetraploid offspring in $B$. napus was reduced $(11 / 22)$. In contrast, DSD events were slightly more frequent in $B$. napus $(20 / 22)$ than in $B$. rapa $(8 / 10)$ and $B$. oleracea $(9 / 11)$. The proportion of TRD events in $M B D$ gene duplication in $B$. napus was similar to that of its two ancestor species. TD and PD were not detected in the three species, suggesting that $M B D$ genes in B. rapa, B. oleracea and B. napus may not be affected by these two events. A study showed that genes increased by WGD were often accompanied by TRD and DSD events [29], which was consistent with what we found in the $M B D$ gene family. TRD may be a long-distance gene transposition through DNAbased or RNA-based transposable elements (TEs) [30]. Therefore, we predicted the TEs within $2000 \mathrm{bp}$ upstream and downstream of the $M B D$ genes (Additional file 2: Table S2). We found 83, 88 and $182 \mathrm{TE}$ 
fragments in B. rapa, B. oleracea and B. napus, respectively. These results showed that the number of TEs of $M B D$ genes, especially DNA transposons, was significantly increased compared with diploid ancestors in $B$. napus. Gene duplication rapidly provides a large number of options for natural selection.

\section{Phylogenetic analysis of $M B D$ genes}

To elucidate the phylogenetic and evolutionary relationships among $M B D$ genes, we constructed a phylogenetic tree based on 74 protein sequences from four dicotyledons (B. rapa, B. oleracea, B. napus and A. thaliana) and two monocotyledons (Oryza sativa and Zea mays,

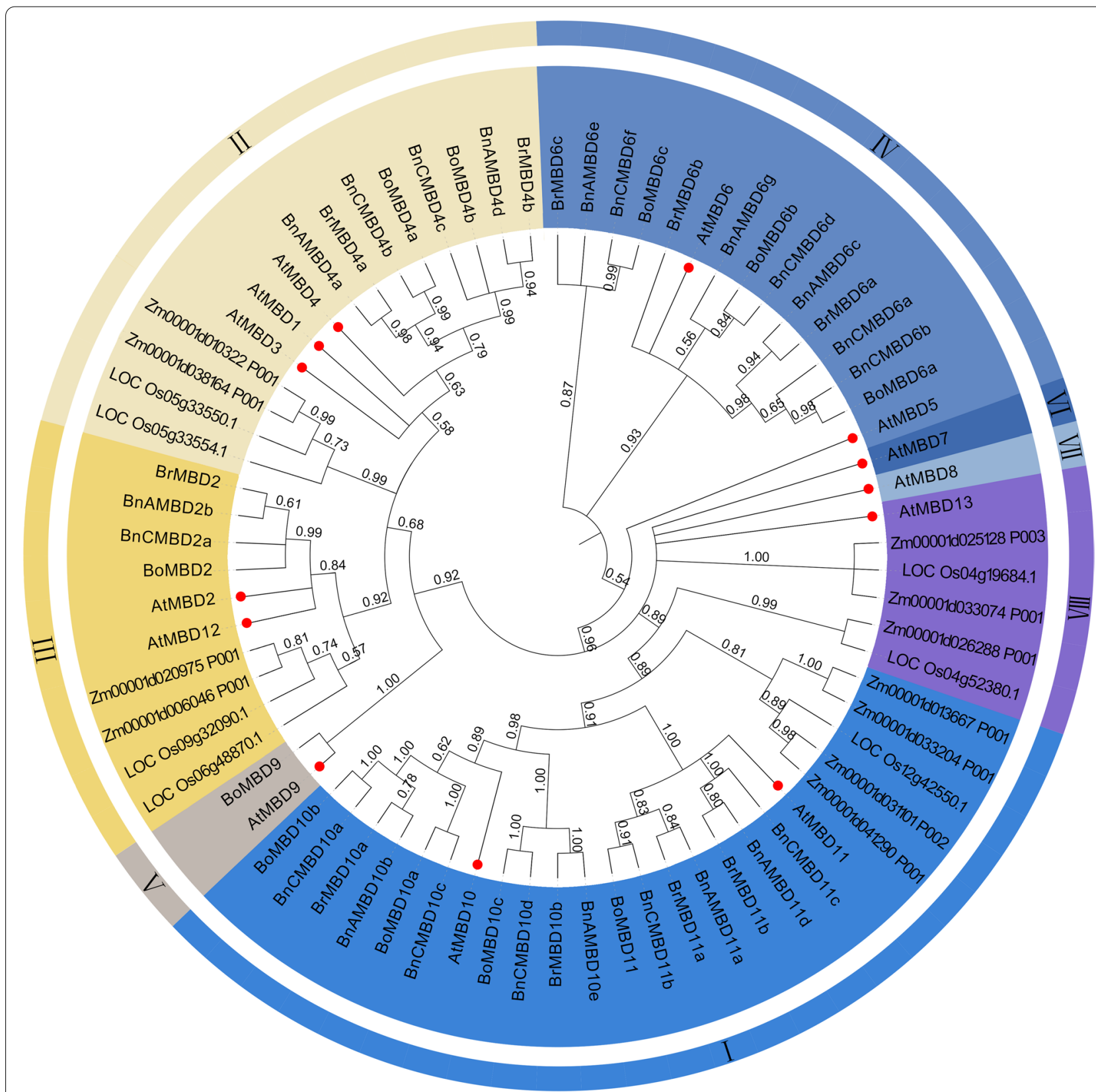

Fig. 2 Phylogenetic tree of $M B D$ gene family in 6 species. MBD genes from four dicotyledons (B. rapa, B. oleracea, B. napus and A. thaliana) and two monocotyledons (Oryza sativa and Zea mays) were displayed in the phylogenetic tree. In the MEGAX software, using the Maximum Likelihood (ML) and 1000 bootstrap repeats, seventy-four protein sequences were analyzed to construct a phylogenetic tree. The phylogenetic tree was divided into 8 clades, and different colors represented different clades. The figure only showed bootstrap values greater than 50\%, and $13 \mathrm{~A}$. thaliana MBD genes were marked with red dots 
Fig. 2). The MBD protein sequences of Arabidopsis were obtained from the TAIR database, and the protein sequences of rice, maize, $B$. rapa, $B$. oleracea and B. napus were identified according to the same criteria. Taking typical monocotyledons and dicotyledons representing Arabidopsis, rice and maize whose $M B D$ gene family had been studied in detail as an outgroup, a phylogenetic tree was constructed together with $B$. rapa, B. oleracea and $B$. napus to make the result more accurate and show the evolutionary direction. Based on the clades of the $M B D$ gene in Arabidopsis, rice and maize, we also divided the phylogenetic tree containing B. rapa, B. oleracea and B. napus into 8 clades, including 24 genes of clade I, 15 genes of clade II, 10 genes of clade III, 15 genes of clade IV, 2 genes of clade V, 1 gene of clade VI, 1 gene of clade VII and 6 genes of clade VIII. Among them, the true $M B D$ genes in Brassica only existed in clades I to V. In this study, $M B D$ genes of monocotyledons, maize and rice, were located in clades I, II, III and VIII, while dicotyledons were distributed in all eight clades. What attracted special attention was that monocotyledons have been confirmed to have no $M B D$ genes in clade IV and clade VI [26]. Monocotyledons and dicotyledons seem to have appeared in the early Cretaceous as the main clades of angiosperms [31]. We found that the $M B D$ genes had a long evolutionary history. As early as the formation period of monocotyledons and dicotyledons, the $M B D$ genes already existed and began to differentiate. In addition, the $M B D$ genes maintained good homology during long evolution among B. rapa, B. oleracea, B. napus, Oryza sativa and Zea mays. $M B D$ genes with homology to the same AtMBD were located in the same clades. However, at the same time, some $M B D$ genes may have lost homologous genes or changed their structure during evolution; for example, AtMBD7 and AtMBD8 lack closely related branches on the phylogenetic tree. Considering their structural integrity, some $M B D$ genes in Oryza sativa, Zea mays, B. rapa, $B$. oleracea and B. napus may not be discussed.

\section{Analysis of $M B D$ genes structure and $G O$ enrichment}

We constructed another phylogenetic tree for the $M B D$ genes of B. rapa, B. oleracea and B. napus and analyzed exon-intron structures to explore the effect of allopolyploidization on the $M B D$ gene structure (Fig. 3). The topological structure of the two phylogenetic trees was consistent, but the analyzed species were not completely

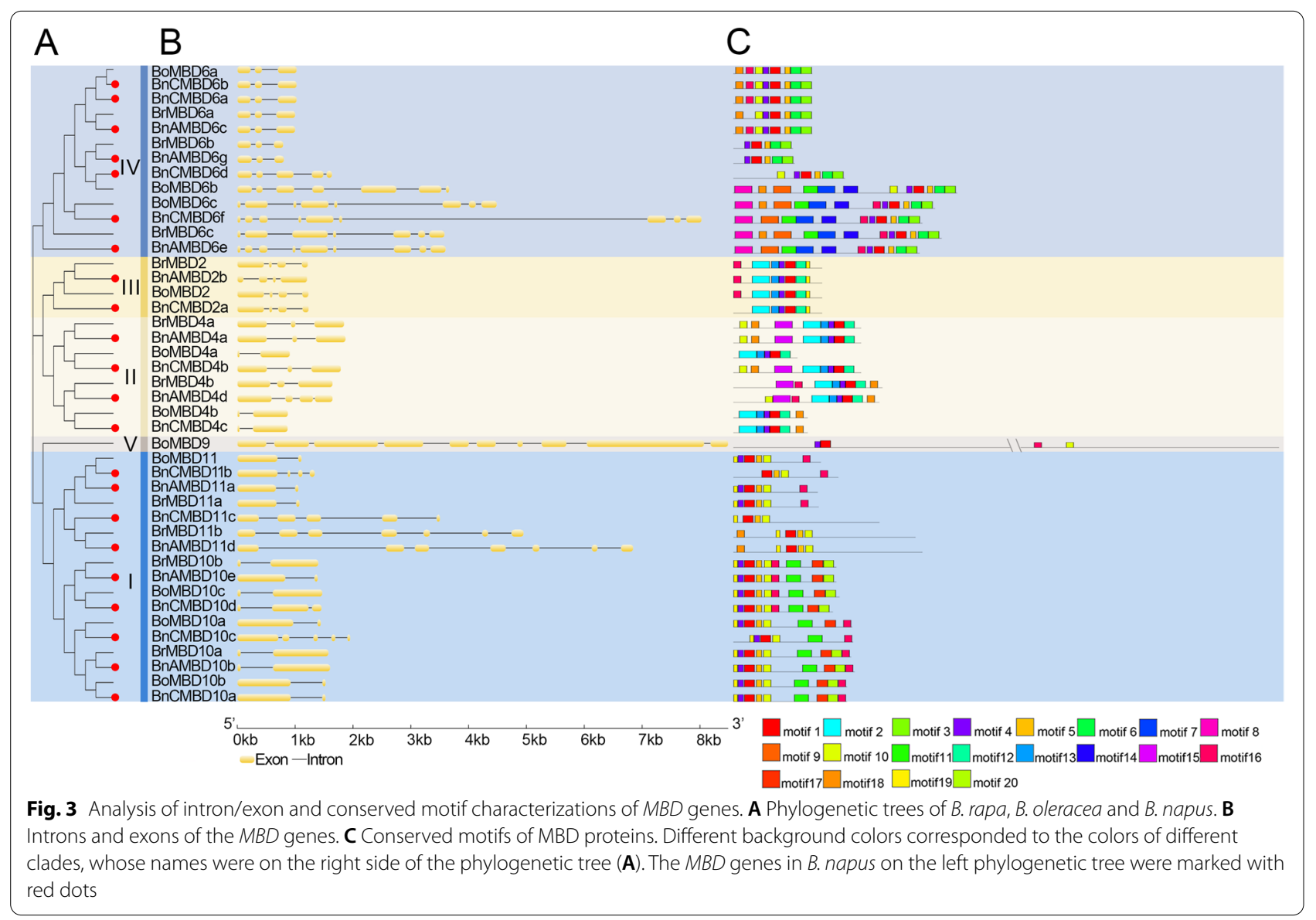


the same. The latter tree only contained members of the Brassica $M B D$ gene family. B. napus and its diploid ancestors did not have corresponding $M B D$ gene family members in clades VI, VII and VIII, so only clades I to $\mathrm{V}$ are shown (Fig. 3A). The same clade used the same background color in both figures and contained the same $M B D$ genes of $B$. rapa, B. oleracea and $B$. napus. The results showed that the number of introns varied significantly among $M B D$ genes, ranging from 1 to 9 (Fig. 3B). Among them, the number of introns in clade II and clade III is relatively small, containing only 1 to 3 introns. We believe that two genes from the terminal branch of the same phylogenetic tree, if one came from $B$. napus and the other from either $B$. rapa or B. oleracea, were considered to have a direct evolutionary relationship. Seventeen out of twenty-two B. napus genes were found to be directly related to their parents. Of the 17 gene pairs, 10 had the same number of introns, and 7 had an increase. The phenomenon of increasing intron numbers was widespread.

To further understand the potential function of $M B D$ genes in B. rapa, B. oleracea and B. napus, we conducted Gene Ontology (GO) enrichment analysis for the three species (Additional file 3: Fig. S1). In B. rapa (Additional file 3: Fig. S1A) and B. oleracea (Additional file 3: Fig. $\mathrm{S} 1 \mathrm{~B}), M B D$ genes were mainly enriched in molecular functions, especially in these three terms: "methyl-CpG binding", "nucleotide binding" and "nucleoside phosphate binding". In B. napus (Additional file 3: Fig. S1C), in addition to being abundantly enriched in the above three molecular functions as in their diploid ancestors, various cellular components represented by "perinucleolar chromocenter" and "nucleolus organizer region" were also enriched.

\section{Prediction of the amino acid sequence and physicochemical properties of the MBD protein}

We also analyzed the conserved motifs of 43 proteins encoded by $M B D$ genes (Fig. 3C). Similarly, most of the clades had similar motif types. However, overall, there was a large gap between the number and type of conserved motifs in the different MBD proteins. Motif 1 and motif 4 existed in almost all MBD proteins. Notably, motif 3 and motif 6 in clade IV were present in every MBD protein and only in this clade. These two motifs may have special functions, distinguishing the proteins of clade IV from the MBD proteins of other clades.

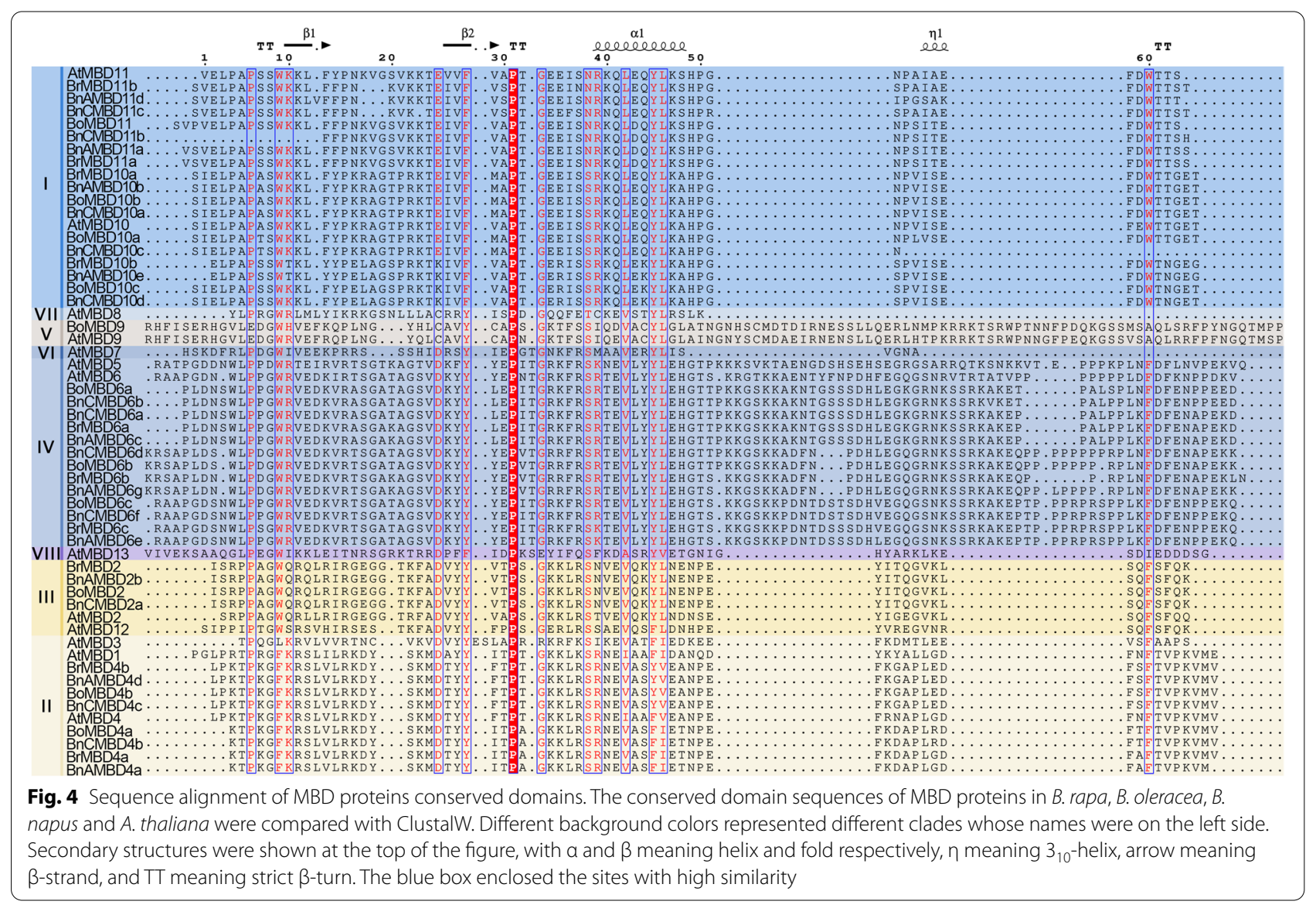


To further understand the structural characteristics of MBD proteins, we compared the domain sequences of B. rapa, B. oleracea, B. napus and A. thaliana (Fig. 4). The blue line framed the sites with high similarity. The top of the figure shows the secondary structure of the protein, and the right side shows the name of the different clades of the phylogenetic tree (Fig. 2). In Figs. 2, 3 and 4, the same background colors represented the same clades. The corresponding clades of Brassica and A. thaliana $M B D$ gene family members in Figs. 2 and 4 remained unchanged. There were multiple MBD domains in AtMBD7, and only the domain sequence with a complete structure is shown in the figure. The MBD domains contained $\alpha$ helix and $\beta$ fold. However, the MBD domain is a hallmark feature of the MBD protein. These results showed that the overall conservation of MBD protein domains was not high, and there were great differences among different clades, but some sites were highly conserved, which may be related to different MBD proteins performing different functions.

We used the ProtPram tool on the ExPASy server to predict the physical and chemical properties of $43 \mathrm{MBD}$ proteins, such as molecular weight (MW), isoelectric point (pI), grand average of hydropathicity (GRAVY) and instability index online (Additional file 4: Table S3). The results showed that among the MBD proteins in $B$. napus, the average protein length (332.4 aa) was lower than that of its diploid ancestors B. rapa (348.3 aa) and $B$. oleracea (500.7 aa). Correspondingly, the average molecular weight of MBD proteins in B. napus was $36.71 \mathrm{kDa}$, which was lower than that of $B$. rapa $(38.62 \mathrm{kDa})$ and $B$. oleracea $(55.31 \mathrm{kDa})$. The $\mathrm{pI}$ of $B$. rapa, B. oleracea and $B$. napus varied from 4.60 to 9.56 , and more than half of them were less than 7. Except for BoMBD10a, the remaining $\mathrm{MBD}$ proteins were all classified as unstable (instability index $>40$ ). Forty-three MBD proteins were all hydrophilic proteins. Using WoLF PSORT software to predict the subcellular location of MBD proteins, the results showed that most $\mathrm{MBD}$ proteins in $B$. napus were located in the same location as their ancestral homologs in the nucleus.

\section{Synteny analysis of $M B D$ genes}

We used the BRAD database to query the syntenic relationship among the $M B D$ genes and to draw a synteny map between $B$. napus and its diploid ancestors (Fig. 5, Additional file 5: Table S4). Two syntenic genes are connected by an arc, with the blue lines linking the between species, and the red lines linking the syntenic paralogs (Fig. 5). A total of 68 pairs of syntenic orthologs and 25 pairs of syntenic paralogs were identified in $B$. napus and its diploid ancestors. Among them, $B n A M B D 10 e$ was located on the unassembled scaffold, so nine syntenic gene pairs associated with it are not shown in the figure. $M B D$ genes of $B$. napus had strong synteny with those of its ancestors. Approximately $72.1 \%$ of $M B D$ gene family members were located in the syntenic region, and syntenic genes were widely distributed in the genomes of the three species. The syntenic genes corresponding to $A t M B D 2$ and $A t M B D 12$ were the same in B. rapa, $B$. oleracea and $B$. napus. Fourteen syntenic gene pairs were found between $B$. rapa and B. oleracea, while only twelve syntenic gene pairs were found between the $A_{n}$ and $\mathrm{C}_{\mathrm{n}}$ subgenomes of $B$. napus, suggesting that $M B D$ syntenic genes loss may occur during allopolyploidization. It is worth mentioning that only AtMBD2, AtMBD4, AtMBD6, AtMBD10, AtMBD11 and AtMBD12 corresponding syntenic genes were found in $B$. rapa, $B$. oleracea and B. napus, while AtMBD1, AtMBD3, AtMBD5, AtMBD7, AtMBD8, AtMBD9 and AtMBD13 were not found. This is probably caused by the loss of some copies of these genes or the changes in their gene structures during the process of evolution, which were not identified as real $M B D$ genes in this study.

We calculated the nonsynonymous (Ka) and synonymous (Ks) substitution values and $\mathrm{Ka} / \mathrm{Ks}$ ratios of duplicated gene pairs in B. napus and its ancestors to characterize the selection pressure on $M B D$ genes during evolution (Table 1). Generally, the $\mathrm{Ka} / \mathrm{Ks}$ ratio is used to represent the selection pressure on genes. A ratio greater than 1 means positive selection, less than 1 means purifying selection, and equal to 1 means neutral selection [32]. The results showed that there were no duplicated gene pairs between $B$. rapa and $B$. oleracea. The $\mathrm{Ka} / \mathrm{Ks}$ ratios of the eight pairs of duplicated genes in the subgenomes of $B$. napus were less than 1 , which indicated that they underwent purifying selection.

\section{Analysis of cis-acting elements in the $M B D$ genes}

Different cis-acting elements in gene promoters may be related to different functions of genes. To identify cisacting elements of $M B D$ genes, gene sequences within $1500 \mathrm{bp}$ upstream of the gene transcription start site (TSS) were extracted and retrieved using PlantCARE [33]. The results showed that the upstream promoter regions of the $M B D$ genes contained a large number of cis-acting elements in B. napus and its diploid ancestors. Figure 6 shows the cis-acting elements associated with four types of life activities: plant development and growth (7), phytohormone responses (11), light responsiveness (24) and stress responses (6). Among them, there were abundant cis-acting elements related to phytohormone responses and light responsiveness, especially the CGTCA-motif and TGACG-motif involved in MeJA-responsiveness, ABRE involved in abscisic acid responsiveness, and the GT1-motif, G-Box, G-box 


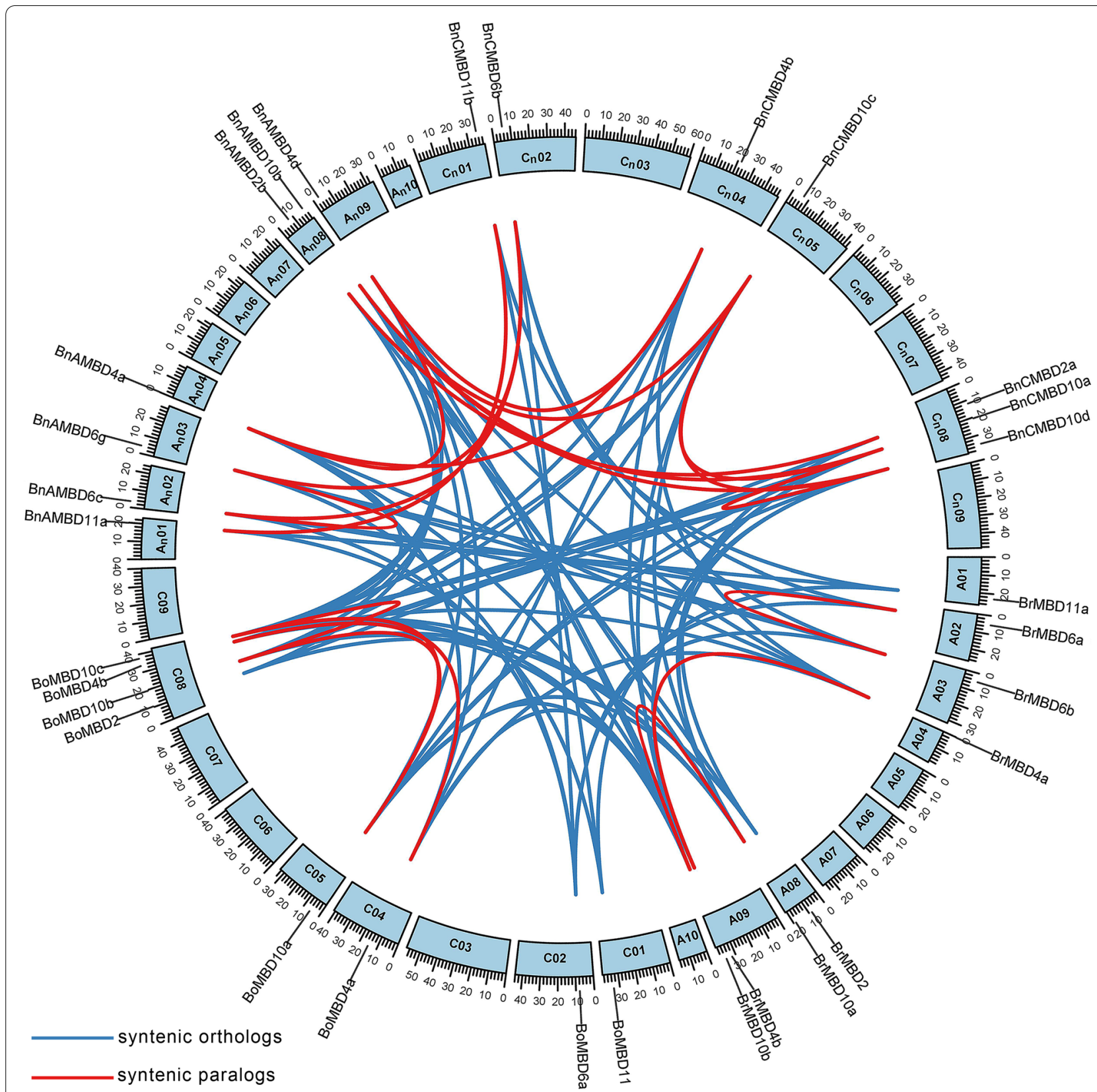

Fig. 5 Genome-wide synteny analysis for $M B D$ genes in B. rapa, B. oleracea and B. napus. B. rapa chromosomes were A01-A10, B. oleracea chromosomes were C01-C09, B. napus $A_{n}$ subgenomic chromosomes were $A_{n} 01-A_{n} 10, B$. napus $C_{n}$ subgenomic chromosomes were $C_{n} 01-C_{n} 09$. All the syntenic genes identified in the MBD genes were mapped to the responding chromosomes, except for the 9 gene pairs that had syntenic relationships with the BnAMBD10e gene located to the unassembled scaffold, which were not shown in the figure. Blue lines linking the syntenic orthologs, and red lines linking the syntenic paralogs

and Box 4, which are related to light responsiveness. A large number of them were found in the identified $M B D$ promoter regions. In addition, ARE related to anaerobic induction made up an important proportion of the stress response elements. The three species all contained a large amount of ARE, and the number in $B$. napus was significantly greater than the sum of its ancestor species. Overall, the number of cis-acting elements found in $B$. napus (517) was roughly the same as the sum found in B. rapa (219) and B. oleracea (294). During the process of allopolyploidization, the number of cis-acting elements related to plant development and growth decreased, but the other three types increased, especially cis-acting elements related to stress, which 
Table $1 \mathrm{Ka} / \mathrm{Ks}$ ratios and selection types of MBD duplicated gene pairs

\begin{tabular}{lllll}
\hline Duplicated gene pairs & Ka & Ks & Ka/Ks & Types of selection \\
\hline BnCMBD2a-BnAMBD2b & 0.009 & 0.059 & 0.146 & Purify selection \\
BnAMBD4a-BnCMBD4b & 0.015 & 0.058 & 0.250 & Purify selection \\
BnCMBD6a-BnCMBD6b & 0.006 & 0.037 & 0.158 & Purify selection \\
BnCMBD6a-BnAMBD6c & 0.012 & 0.037 & 0.319 & Purify selection \\
BnCMBD6b-BnAMBD6c & 0.018 & 0.075 & 0.234 & Purify selection \\
BnAMBD6e-BnCMBD6f & 0.010 & 0.065 & 0.155 & Purify selection \\
BnCMBD10a-BnAMBD10b & 0.039 & 0.143 & 0.270 & Purify selection \\
BnCMBD10d-BnAMBD10e & 0.042 & 0.067 & 0.626 & Purify selection \\
\hline
\end{tabular}

may be related to the stronger resilience of tetraploid plants.

\section{Gene expression pattern analysis of $M B D$ genes}

We used flowers, leaves, siliques and stems to analyze the expression patterns of $M B D$ genes in the three species (Fig. 7, Additional file 6: Table S5). Overall, although there was a significant difference in expression among the genes, the specificity between tissues was weak. However, the expression of different genes between tissues still had a certain preference. For example, the expression level of $B n C M B D 10 c$ in leaves was significantly higher than that of the other three tissues, but the expression

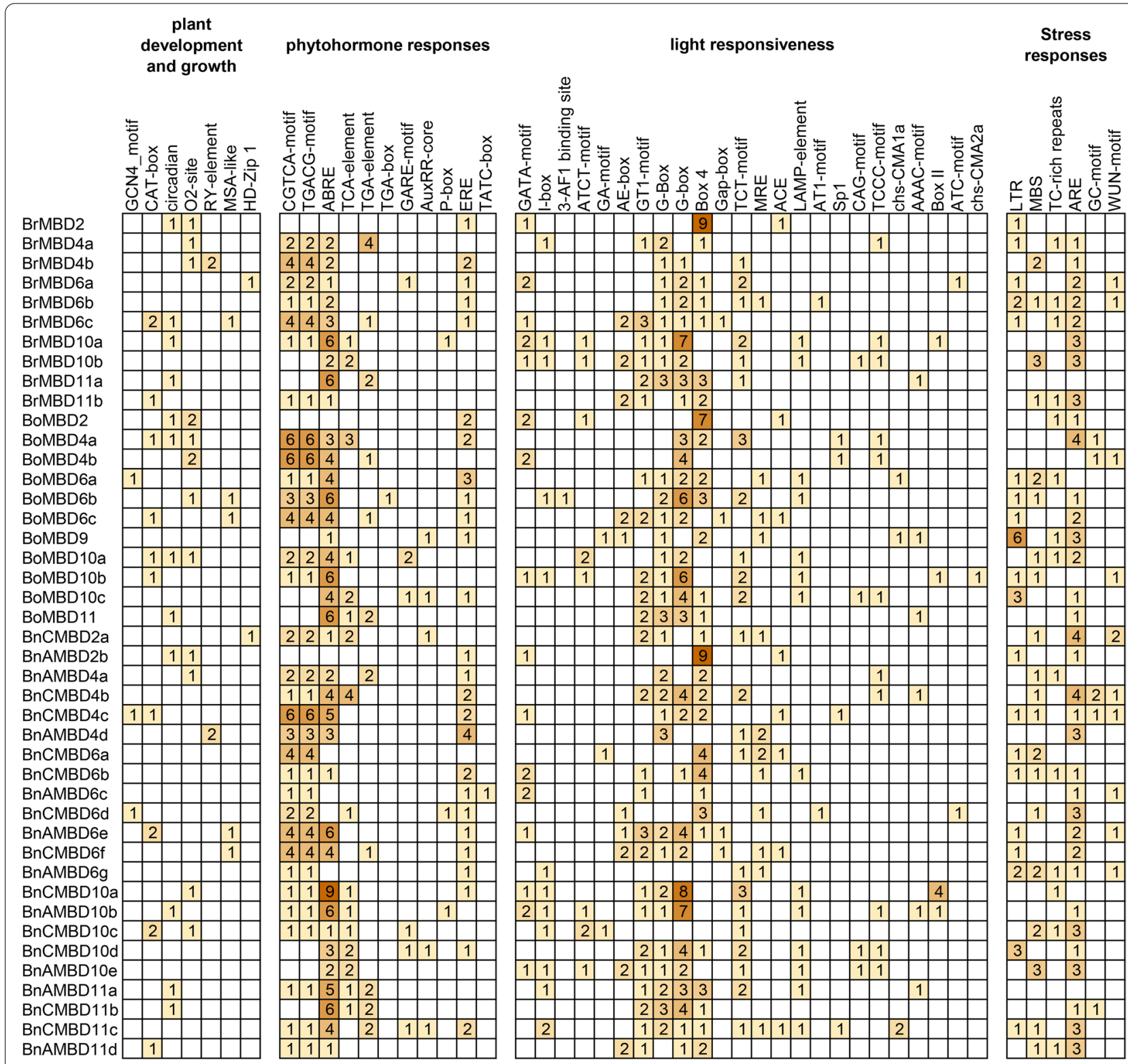

Fig. 6 Cis-acting elements in the promoter regions of the MBD genes 


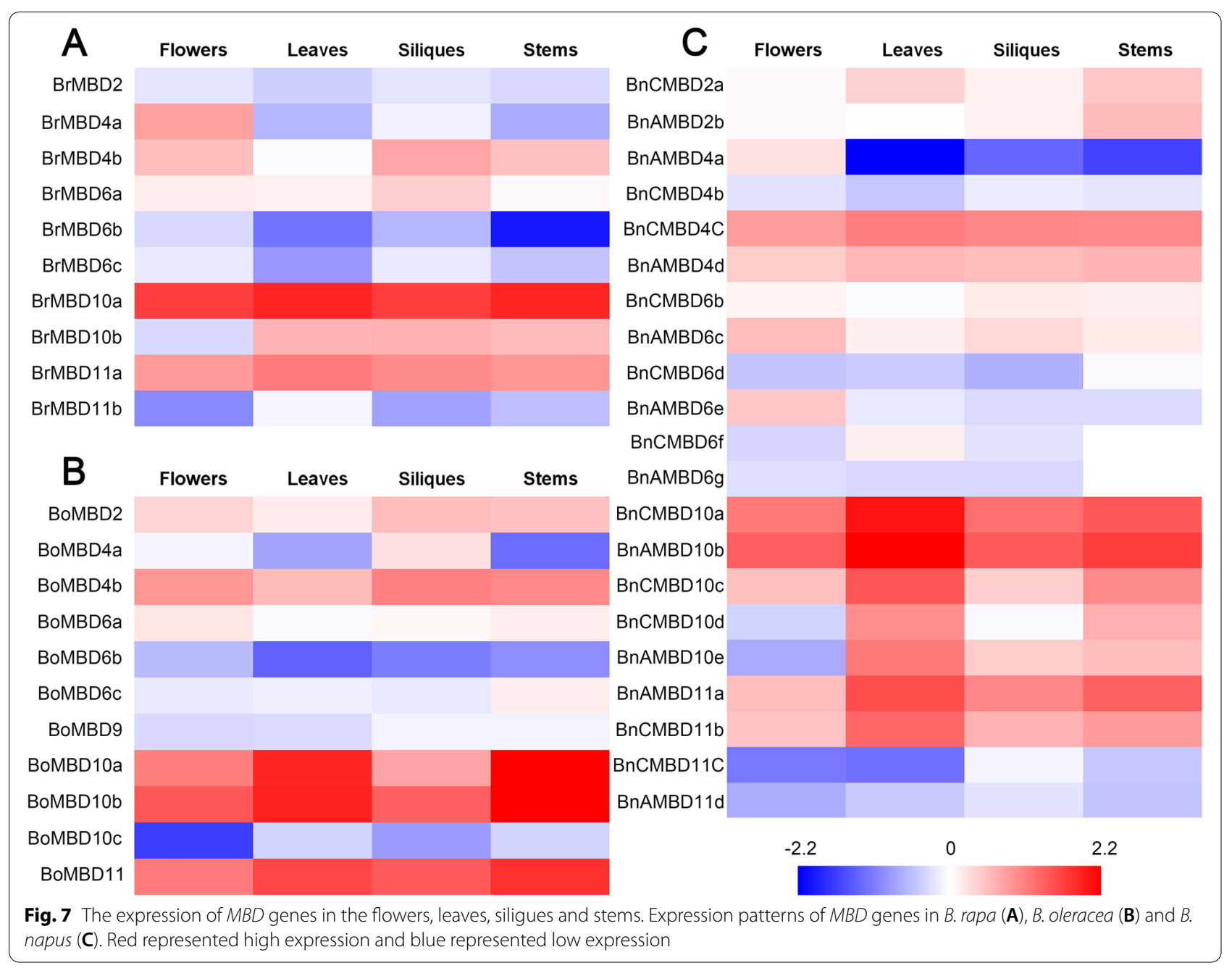

of $B n C M B D 11 c$ was the opposite. The number of clade I genes (Fig. 2) of all three species (except BrMBD11b, $B o M B D 10 c, B n C M B D 11 c$ and BnAMBD11d) showed high expression levels. In contrast, the gene expression levels of clade IV were relatively low in all species, and $B n C M B D 6 a$ was not expressed in any of the four tissues. The difference in expression levels between different clades may be a manifestation of the diverse functions of $M B D$ genes.

To explore whether the expression patterns of $M B D$ genes changed among the four tissues during allopolyploidization, we compared the expression patterns between gene pairs with direct evolutionary relationships (Fig. 3A). The expression patterns of most gene pairs changed. For example, $B r M B D 2$ was expressed at a low level in the four tissues, while $B n A M B D 2 b$ was expressed at a high level in the stems. BoMBD4a was highly expressed in siliques and low in leaves and stems, but the expression level of $B n C M B D 4 b$ was almost the same among the four tissues. BoMBD10c had low expression levels in the four tissues, especially in flowers. $B n C M B D 10 d$ expression was low in flowers, but it had high expression levels in leaves and stems. Interestingly, only one of the eight pairs of duplicated genes (Table 1) shared the same expression pattern. This may further indicate that functional differentiation of the $M B D$ genes occurred during evolution.

We used FPKM (fragments per kilobase million) values to analyze the expression bias of 17 gene pairs with direct evolutionary relationships (Additional file 7: Table S6). Because the homologous genes of $M B D 2$ existed in only one parent, it was not discussed. Different genes had different expression biases in tissues. The expression of $M B D 4$ in leaves was biased toward B. rapa and biased toward B. oleracea in the other three tissues. MBD6 expression in leaves and siliques was biased toward $B$. oleracea, and it was biased toward B. rapa in flowers and stems. The expression of $M B D 10$ was biased toward $B$. oleracea in flowers and siliques and toward $B$. rapa in leaves and stems. The expression of MBD11 was biased 
to $B$. rapa in all four tissues. Overall, for $M B D$ genes with expression bias, most genes were biased toward $B$. oleracea in siliques and $B$. rapa in leaves and stems, while the number of genes biased toward B. rapa and B. oleracea in flowers was the same.

\section{Discussion}

With the significant increase in the number of genomesequenced plants, the understanding of polyploidy evolution has moved from a "dead end" to today's "contemporary innovation" [34]. Polyploidy is common in angiosperms, and WGD events contribute to the dominance of angiosperms by providing important genes for seed and flower development [35]. Some gene families also change during this process, such as family expansion or contraction [36]. In Brassica, we also observed an expansion of this gene family, with more $M B D$ genes identified in $B$. napus than in any other species previously, such as Arabidopsis, rice, maize, and wheat. The Brassica genus contains neotetraploidization and mesohexaploidization species and is an excellent model for studying polyploidy evolution [37]. The MBD protein participates in the DNA methylation process and is closely related to plant growth and development. The $M B D$ gene family has been studied in Arabidopsis, rice, maize, and wheat [26, 38], but studies in Brassica have not yet been reported. We analyzed the $M B D$ gene family in $B$. rapa, B. oleracea, and $B$. napus to understand the influence of allopolyploidization on this gene family.

\section{$M B D$ gene sequence differentiation is not achieved overnight but gradually evolves}

From the perspective of phylogenetic evolution, $M B D$ genes of Arabidopsis, rice and maize can be divided into eight clades, each with its unique characteristics [26]. Arabidopsis $M B D$ gene family members were derived from previous studies, so we did not delete even potential $M B D$ genes. However, the five species reidentified in this study, B. rapa, B. oleracea, B. napus, Oryza sativa and Zea mays, were identified based on strict criteria. $M B D$ genes were discussed only if the encoded MBD proteins contained a complete domain (Fig. 2). Therefore, the number of genes encoding the MBD protein of rice and maize was less than 17 and 14, respectively [39], in phylogenetic tree construction. In B. rapa, B. oleracea, and $B$. napus, $M B D$ gene family members were identified in only five clades of the phylogenetic tree, lacking three clades compared with Arabidopsis $M B D$ gene family members in eight clades. This may be due to the loose identification criteria of Arabidopsis, used for constructing clades of potential $M B D$ genes. After Brassica and Arabidopsis separated, some proteins encoded by the $M B D$ genes in
B. rapa, $B$. oleracea, and $B$. napus may be incomplete or lost domains during the process of independent evolution. Monocotyledons and dicotyledons are two aspects of the angiosperms. Clade IV and VI genes are unique to dicotyledons and are not found in rice and maize. This may indicate that clade IV and clade VI of $M B D$ genes evolved independently in dicotyledon clades. Although most of the genes in each clade of the $M B D$ gene family are related to DNA methylation and demethylation, only the proteins encoded by these two clades can still specifically bind to methylation sites in vitro [40]. The methylbinding ability of MBD proteins is not achieved overnight but gradually in evolves. The remaining clade genes have much older evolutionary histories and may have more traditional functions during plant growth and development, although they have not been verified to have binding activity in vitro.

Syntenic gene pairs share the same ancestral genomic fragments, and they often have certain similarities in function, so the analysis of syntenic genes is very important for gene and genome research [24]. Syntenic and collinear gene pairs can be identified by searching for conserved blocks, allowing us to better understand the origin of genes. We found that $M B D$ genes had great synteny. Syntenic gene pairs may indicate that $M B D$ genes have different conservation levels during evolution. The syntenic genes corresponding to AtMBD2, AtMBD4, AtMBD6, AtMBD10, AtMBD11 and AtMBD12 may be more conserved and retain more features of their ancestral genes. Some genes with a weak relationship with synteny may be due to complete transposition and a loss of ancestors, or they may be ancient genes and not closely related to the others [28]. For the duplicated gene pairs with direct evolutionary relationships, we calculated the $\mathrm{Ka} / \mathrm{Ks}$ values to evaluate the selection type (Table 1 ). After allopolyploidization, the $\mathrm{Ka} / \mathrm{Ks}$ ratio between $M B D$ genes in $B$. napus was less than 1 , and the $M B D$ genes were under strong purifying selection pressure. Purifying selection may restrict the spread of alleles or mutations so that most $M B D$ genes can still retain the characteristics of their ancestor genes [41], which also verified their strong synteny. These genes still retained multiple pairs of duplicate genes in this case, which also proved that polyploidy may protect genes through rapid subfunctionalization [42]. This phenomenon has also been found in another gene family in Brassica [43].

In terms of gene structure, introns interrupt multiple exon combination sequences and generate mature mRNA through shearing [44]. In our study, the $M B D$ genes were less conserved, and the phenomenon of intron acquisition generally occurred. Among the 17 pairs of genes with a direct evolutionary relationship, the number of introns in 10 pairs of genes remained unchanged, and 7 pairs of 
genes increased, while no intron reduction was found (Fig. 3B). Some researchers believe that introns provide advantages to plants, such as regulating gene expression and increasing protein diversity [45]. The phenomenon of intron increase has also appeared in other gene families in B. napus [46], indicating that the process of allopolyploidization may enhance the ability of plants to adapt to the environment through an increase in gene introns.

\section{Allopolyploidization increases the diversity of MBD protein motifs}

The types of MBD protein motifs are abundant, but there are great differences between different clades (Fig. 3C). Motif 1 and motif 4 were highly conserved among MBD proteins and may be characteristic motifs of MBD proteins. Motif 3 and 6 were unique in clade IV and may be related to the specific function of clade IV. Similar phenomena were observed in the potato $M A T E$ gene family [47]. The GO enrichment results showed that $M B D$ genes were mostly enriched in "methyl-CpG binding", "nucleotide binding" and "nucleoside phosphate binding", which was consistent with the known function of the $M B D$ gene family. However, the difference in motifs among different clades, especially the existence of unique motifs in clades such as motif 3 and motif 6, may indicate functional divergence among $M B D$ genes. Further analysis of the amino acid sequences showed that the MBD protein domains were less conserved, except for individual sites, but the protein within the same clade was highly conserved (Fig. 4). This may be caused by the process that endowed multicopy genes with different fates, in which many genes return to a single copy after extensive fractionation of polyploidy expanded homologous genes [2]. The conservation of the same clade among different species suggests that the $M B D$ gene sequences may differentiate as early as the formation period of monocotyledons and dicotyledons, and further consolidate during polyploidy. In addition, the motifs of the proteins encoded by seven gene pairs with direct evolutionary relationships changed, although there was no one-to-one correspondence with gene pairs in which the intron increased. Compared with diploid ancestors, the numbers of these seven MBD protein motifs increased or decreased and the motif types changed in $B$. napus. This indicated that allopolyploidization also led to $M B D$ gene sequencing and functional differentiation.

\section{$M B D$ gene expression bias in allopolyploid $B$. napus may be regulated by TEs and cis-acting elements}

Ancestral species usually provide two or more asymmetric subgenomes for allopolyploids [48]. The contribution of both parents to gene expression is not completely additive, and gene expression dominance has become a characteristic feature of many allopolyploid plants [49].
Homologous expression bias between subgenomes can promote the adaptability and flexibility of newly formed polyploids to the environment $[50,51]$. In our study, the subgenomes of $A_{n}$ and $C_{n}$ retained the structure of ancestral chromosomes to some extent, and the position and number of the $M B D$ genes on chromosomes is mostly unchanged (Fig. 1). However, the expression of some $M B D$ genes is biased among multiple tissues (Additional file 7: Table S6), and more than half of the genes were biased in $B$. rapa, slightly more than $B$. oleracea.

The expression pattern of $M B D$ genes changed significantly in Brassica during the allopolyploidization process. Cis-acting elements and TEs can regulate gene expression and change the gene expression patterns [52, 53]. We divided the cis-acting elements of $M B D$ genes into four groups: plant development and growth, phytohormone responses, light responsiveness and stress responses. The number of cis-acting elements in $B$. oleracea was greater than that in $B$. rapa, and the number of cis-acting elements in B. napus was almost the same as the sum of the diploid ancestors. Interestingly, while the total amount remained the same, there were increases or decreases in different groups. B. napus may enhance its adaptability to the environment through the regulation of cis-acting elements. On the other hand, TE amplification may have driven the formation of the $B$. napus genome [54]. We identified 83, 88 and 182 TE fragments within $2000 \mathrm{bp}$ upstream and downstream of the $M B D$ gene in the $B$. rapa, $B$. olerace $a$ and $B$. napus genomes, respectively. Compared with the diploid ancestors, the number of TE fragments in B. napus increased significantly, which may be activated by polyploidization [55]. Gene expression was affected by the number and distance of nearby TEs [56]. The subgenome with the greatest TE density had a relatively high probability of gene loss and inactivation and a weak overall expression level [57]. The number of TEs in B. oleracea was slightly greater than that in $B$. rapa, which may also provide an explanation for the bias of $M B D$ gene expression in B. napus.

\section{Conclusions}

In this study, 10, 11 and $22 M B D$ genes were identified in $B$. rapa, $B$. oleracea and $B$. napus, respectively. The WGD, TRD and DSD events maintained the number of $M B D$ gene family members in these three species, and there was a strong syntenic relationship among these genes. The $\mathrm{Ka} / \mathrm{Ks}$ ratios of duplicated genes were less than 1 , and intense purification pressure may promote $M B D$ genes to retain genes through sequence and functional differentiation. Sequence differentiation of $M B D$ genes has been occurring continuously since the formation period of monocotyledons and dicotyledons, and each of the eight clades has its own characteristics. During the process of 
allopolyploidization, the $M B D$ genes retained their original structural features and also showed some new changes, such as an increase in intron number in B. napus. Meanwhile, MBD proteins in different clades had their own motifs, and the number and type of motifs changed during the allopolyploidization process. This process may increase the number of cis-acting elements and activate TEs. In B. napus, cis-acting elements and TEs may regulate the expression of $M B D$ genes to improve the plant's ability to adapt to the environment. At the same time, their number imbalance in the $A_{n}$ and $C_{n}$ subgenomes may lead to $M B D$ gene expression bias in $B$. napus. These results can enhance the understanding of the $M B D$ gene family and provide a reference for future exploration of allopolyploids.

\section{Materials and methods}

\section{Identification of $M B D$ genes}

In this study, thirteen potential $A$. thaliana MBD protein sequences were obtained from TAIR database (https://www. arabidopsis.org/) and used as query sequences for BLASTp searches (E-value $<10^{-5}$ ) of proteins in B. rapa, B. oleracea and B. napus in the BRAD database (http://brassicadb. $\mathrm{cn} / \# /)$ [58]. To identify the true MBD proteins, only the proteins containing the complete MBD domain were identified as $\mathrm{MBD}$ proteins. After the repetitive values were removed, all the identified candidate protein sequences were confirmed by CDD (https://www.ncbi.nlm.nih.gov/Structure/ cdd/wrpsb.cgi) [59], Pfam (http://pfam.xfam.org/) [60] and InterPro (https://www.ebi.ac.uk/interpro/search/seque nce/) [61] databases, and the proteins with complete MBD domains that could be queried in all three databases were retained. Finally, the identified $M B D$ genes were named based on their homology with $M B D$ genes in A. thaliana. Using the same standards and methods, the MBD proteins in Oryza sativa and Zea mays were identified in the public database Phytozome (https://phytozome-next.jgi.doe.gov/), and the required genomic data were obtained [62].

\section{Characterization of the MBD proteins}

Physical and chemical properties of MBD proteins such as molecular weight (MW), grand average of hydropathicity (GRAVY), instability index and isoelectric point (pI) were predicted online using ProtPram tool on ExPASy server (https://www.expasy.org/) [63].The WoLF PSORT (https://wolfpsort.hgc.jp/) [64] online tool predicts subcellular localization of MBD proteins.

\section{Chromosome location and gene structure analysis}

The location information of $M B D$ genes of B. rapa, B. oleracea and $B$. napus were obtained from BRAD database, and the gene chromosome location diagram was drawn by MapInspect software. The coding sequences (CDS) were analyzed on the Gene Structure Display Server (GSDS)
2.0 (http://gsds.gao-lab.org/) [65], and the exon/intron structures of the $M B D$ genes were displayed.

\section{Identification of TEs and cis-acting elements}

The $1500 \mathrm{bp}$ upstream sequences of $M B D$ genes were extracted from BRAD database, and Plant Cis-Acting Regulatory Element (PlantCARE) website (http://bioin formatics.psb.ugent.be/webtools/plantcare/html/) was used to search and analysis cis-acting elements in promoter regions and classified them according to their functions [33]. TEs within $2000 \mathrm{bp}$ upstream and downstream of $M B D$ genes were predicted in database Repbase Update (RU, https://www.girinst.org/) [66].

\section{Gene duplication and synteny analysis}

The online database Plant Duplicate Gene Database (http:// pdgd.njau.edu.cn:8080/) was used to query information about five replication modes [67]. At the same time, (a) sequence coverage length $>80 \%$; (b) alignment region identification $>80 \%$ as the standard to identify duplicated genes [68]. In order to further explore the evolutionary pressure, DnaSP software was used to calculate the $\mathrm{Ka}$, $\mathrm{Ks}$ and $\mathrm{Ka} / \mathrm{Ks}$ values of the identified duplicated gene pairs [69]. The syntenic genes information of B. rapa, B. oleracea, B. napus and A. thaliana were queried in BRAD database [24], and the circos diagram was drawn using TBtools to express the syntenic relationships among B. rapa, B. oleracea and B. napus [70].

\section{Phylogenetic relationship analysis}

We collected MBD protein sequences of four dicotyledons (B. rapa, B. oleracea, B. napus and A. thaliana) and two monocotyledons (Oryza sativa and Zea mays). Using ClustalX to perform multiple sequence alignments of their sequences, MEGA X used the alignment results to construct a Maximum Likelihood (ML) tree with 1000 bootstrap repeats [71]. The Interactive Tree of Life (https://itol.embl. $\mathrm{de} /$ ) was the final beautification of the phylogenetic tree [72].

\section{Conserved motifs and MBD domains analysis}

The 20 motifs of $B$. napus and its ancestors MBD proteins were searched on the online website The MEME Suite (https://meme-suite.org/meme/) [73]. Easy Sequencing in PostScript (ESPript) 3.0 (https://espript.ibcp.fr/ESPript/ ESPript/index.php) [74] was used to draw the conserved domains map, which the sequence data of MBD proteins in B. rapa, B. oleracea, B. napus and $A$. thaliana were collected from Pfam database and compared by ClustalW.

\section{Gene Ontology enrichment analysis}

To predict the potential functions of genes and understand biological pathways, TBtools was used to perform Gene Ontology (GO) enrichment analysis of $M B D$ genes in B. rapa, B. oleracea and B. napus [70]. 


\section{Analysis of $M B D$ genes expression in stems, leaves, flowers and siliques}

B. napus (cv. Darmor) and its diploid ancestors, B. rapa (cv. Chiifu) and B. oleracea (cv. Jinzaosheng), were planted in natural environment in Wuhan, China, and the seeds came from the Oil Crops Research Institute, Chinese Academy of Agricultural Sciences. Plant materials were collected and studied with institutional permission and in accordance with national standards. The stems, leaves, flowers, and siliques (10 days after pollination) of healthy 6-month-old plants were collected and immediately frozen in liquid nitrogen. $M B D$ gene expression patterns in four tissues were analyzed using Illumina RNA-seq data [75]. After normalization of FPKM values, HemI software was used to present heatmap [76].

\begin{abstract}
Abbreviations
AA: A. thaliana: Arabidopsis thaliana; B. rapa: Brassica rapa; B. oleracea: Brassica oleracea; B. napus: Brassica napus; BLAST: Basic Local Alignment Search Tool; DSD: Dispersed duplication; CDS: Coding sequences; ESPript: Easy Sequencing in PostScript; FPKM: Fragments per kilobase million; GRAVY: Grand average of hydropathicity; GSDS: Gene Structure Display Server; ISWI: Imitation SWItch; Ka: Non-synonymous substitution; KDa: kilodaltons; Ks: Synonymous substitution; LF: Least fractionated blocks; MBD: Methyl-CpG-Binding Domain; MF1: Medium fractionated blocks; MF2: Most fractionated blocks; ML: Maximum Likelihood; MW: Molecular weight; PD: Proximal duplication; PI: Isoelectric point; PlantCARE: Plant Cis-Acting Regulatory Element; RU: Repbase Update; SRA: SET and RING finger-associated domain; TD: Tandem duplication; TRD: Transposed duplication; TSS: Transcription start site; WGD: Whole genome duplication; WGT: Whole-genome triplication.
\end{abstract}

\section{Supplementary Information}

The online version contains supplementary material available at https://doi. org/10.1186/s12870-022-03485-0.

Additional file 1: Table S1. The five duplicated types of $M B D$ genes in $B$. napus and its ancestors.

Additional file 2: Table S2. The upstream and downstream transposable elements of $M B D$ genes.

Additional file 3: Figure S1. GO enrichment analysis of $B$. rapa (A), B. oleracea (B) and B. napus (C). Blue is molecular function, red is cellular component.

Additional file 4: Table S3. MBD family member characteristics and subcellular localization.

Additional file 5: Table S4. Synteny analysis of MBD genes in B. rapa, $B$. oleracea, B. napus and A. thaliana.

Additional file 6: Table S5. FPKM values of MBD genes in B. rapa, $B$. olerace $a$ and B. napus.

Additional file 7: Table S6. The $\| \log _{2} \mathrm{FC}$ of $M B D$ genes in four tissues.

\section{Acknowledgments}

This study was supported by the National Natural Science Foundation of China (31970241)

\section{Authors' contributions}

Y.X. and J.W. conceived and designed this study. Y.X. conducted analysis and wrote the manuscript. M.L. contributed the sequencing data of gene expression. J.W. revised and edited the manuscript. All authors have read and agreed to the published version of the manuscript.
Funding

This study was supported by the National Natural Science Foundation of China (31970241).

\section{Availability of data and materials}

The datasets supporting the conclusions of this article are included within the article and its additional files. The raw RNA-seg data of the flowers, leaves, siliques and stems of B. rapa, B. oleracea and B. napus came from the NCBI database (https://www.ncbi.nlm.nih.gov//bioproject/489323), with the accession number (SRR7816633-SRR7816668). Public access to the database is open.

\section{Declarations}

Ethics approval and consent to participate

Not applicable.

Consent for publication

Not applicable.

Competing interests

The authors declare that they have no competing interests.

\section{Author details}

${ }^{1}$ State Key Laboratory of Hybrid Rice, College of Life Sciences, Wuhan University, Wuhan 430072, China. ${ }^{2}$ Key Laboratory of Resource Biology and Biotechnology in Western China, Ministry of Education, College of Life Sciences, Northwest University, Xi'an 710069, China.

Received: 29 October 2021 Accepted: 21 February 2022

Published online: 07 March 2022

\section{References}

1. Van de Peer Y, Mizrachi E, Marchal K. The evolutionary significance of polyploidy. Nat Rev Genet. 2017;18(7):411-24.

2. Zhang K, Wang X, Cheng F. Plant polyploidy: origin, evolution, and its influence on crop domestication. Horticult Plant J. 2019;5(6):231-9.

3. Wu Y, Lin F, Zhou Y, Wang J, Sun S, Wang B, et al. Genomic mosaicism due to homoeologous exchange generates extensive phenotypic diversity in nascent allopolyploids. Natl Sci Rev. 2021:8(5):nwaa277.

4. Cai X, Chang L, Zhang T, Chen H, Zhang L, Lin R, et al. Impacts of allopolyploidization and structural variation on intraspecific diversification in Brassica rapa. Genome Biol. 2021;22(1):166

5. Mandakova T, Lysak MA. Post-polyploid diploidization and diversification through dysploid changes. Curr Opin Plant Biol. 2018;42:55-65.

6. Tayale A, Parisod C. Natural pathways to polyploidy in plants and consequences for genome reorganization. Cytogenet Genome Res. 2013;140(2-4):79-96.

7. Wen J, Li PF, Ran F, Guo PC, Zhu JT, Yang J, et al. Genome-wide characterization, expression analyses, and functional prediction of the NPF family in Brassica napus. BMC Genomics. 2020;21(1):871.

8. Mhiri C, Parisod C, Daniel J, Petit M, Lim KY, Dorlhac de Borne F, et al. Parental transposable element loads influence their dynamics in young Nicotiana hybrids and allotetraploids. New Phytol. 2019;221(3):1619-33.

9. Edger PP, Heidel-Fischer HM, Bekaert M, Rota J, Glockner G, Platts AE, et al. The butterfly plant arms-race escalated by gene and genome duplications. Proc Natl Acad Sci U S A. 2015;112(27):8362-6.

10. Salman-Minkov A, Sabath N, Mayrose I. Whole-genome duplication as a key factor in crop domestication. Nat Plants. 2016;2:16115.

11. Zhang H, Lang Z, Zhu JK. Dynamics and function of DNA methylation in plants. Nat Rev Mol Cell Biol. 2018;19(8):489-506.

12. Liu Y, Zhang X, Blumenthal RM, Cheng X. A common mode of recognition for methylated CpG. Trends Biochem Sci. 2013;38(4):177-83.

13. Ichino L, Boone BA, Strauskulage L, Harris CJ, Kaur G, Gladstone MA, et al. MBD5 and MBD6 couple DNA methylation to gene silencing through the J-domain protein SILENZIO. Science. 2021;372(6549):1434-9.

14. Wang CL, Dong XM, Jin D, Zhao YS, Xie SJ, Li XJ, et al. Methyl-CpG-binding domain protein MBD7 is required for active DNA demethylation in Arabidopsis. Plant Physiol. 2015;167(3):905-14. 
15. Potok ME, Wang Y, Xu L, Zhong Z, Liu W, Feng S, et al. Arabidopsis SWR1associated protein methyl-CpG-binding domain 9 is required for histone H2A.Z deposition. Nat Commun. 2019;10(1):3352.

16. Nie WF, Lei M, Zhang M, Tang K, Huang H, Zhang C, et al. Histone acetylation recruits the SWR1 complex to regulate active DNA demethylation in Arabidopsis. Proc Natl Acad Sci U S A. 2019;116(33):16641-50.

17. Scheid R, Chen J, Zhong X. Biological role and mechanism of chromatin readers in plants. Curr Opin Plant Biol. 2021;61:102008.

18. Qu M, Zhang Z, Liang T, Niu P, Wu M, Chi W, et al. Overexpression of a methyl-CpG-binding protein gene OsMBD707 leads to larger tiller angles and reduced photoperiod sensitivity in rice. BMC Plant Biol. 2021;21(1):100.

19. Gigek CO, Chen ES, Smith MA. Methyl-CpG-binding protein (MBD) family: epigenomic read-outs functions and roles in tumorigenesis and psychiatric diseases. J Cell Biochem. 2016;117(1):29-38.

20. Wang Y, Zhang L, Wu GR, Zhou Q, Yue HH, Rao LZ, et al. MBD2 serves as a viable target against pulmonary fibrosis by inhibiting macrophage $\mathrm{M} 2$ program. Sci Adv. 2021;7(1):eabb6075.

21. Song X, Wei Y, Xiao D, Gong K, Sun P, Ren Y, et al. Brassica carinata genome characterization clarifies U's triangle model of evolution and polyploidy in Brassica. Plant Physiol. 2021;186(1):388-406.

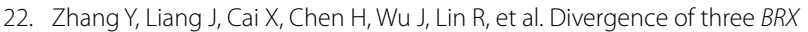
homoeologs in Brassica rapa and its effect on leaf morphology. Hortic Res. 2021;8(1):68.

23. Cheng F, Wu J, Fang L, Sun S, Liu B, Lin K, et al. Biased gene fractionation and dominant gene expression among the subgenomes of Brassica rapa. PLoS One. 2012;7(5):e36442.

24. Cheng F, Wu J, Fang L, Wang X. Syntenic gene analysis between Brassica rapa and other Brassicaceae species. Front Plant Sci. 2012;3:198.

25. Hu D, Jing J, Snowdon RJ, Mason AS, Shen J, Meng J, et al. Exploring the gene pool of Brassica napus by genomics-based approaches. Plant Biotechnol J. 2021;19(9):1693-712.

26. Springer NM, Kaeppler SM. Evolutionary divergence of monocot and dicot methyl-CpG-binding domain proteins. Plant Physiol. 2005;138(1):92-104.

27. Cheng F, Mandakova T, Wu J, Xie Q, Lysak MA, Wang X. Deciphering the diploid ancestral genome of the mesohexaploid Brassica rapa. Plant Cell. 2013;25(5):1541-54

28. Cao Y, Han Y, Meng D, Abdullah M, Yu J, Li D, et al. Expansion and evolutionary patterns of GDSL-type esterases/lipases in Rosaceae genomes. Funct Integr Genomics. 2018;18(6):673-84.

29. Wang Y, Ficklin SP, Wang X, Feltus FA, Paterson AH. Large-scale gene relocations following an ancient genome triplication associated with the diversification of core eudicots. PLoS One. 2016;11(5):e0155637.

30. Wang Y, Wang X, Paterson AH. Genome and gene duplications and gene expression divergence: a view from plants. Ann NY Acad Sci. 2012;1256:1-14

31. Coiffard C, Kardjilov N, Manke I, Bernardes-de-Oliveira MEC. Fossil evidence of core monocots in the early cretaceous. Nat Plants. 2019;5(7):691-6.

32. Zhang H, Li S, Shi M, Wang S, Shi L, Xu F, et al. Genome-wide systematic characterization of the NPF family genes and their transcriptional responses to multiple nutrient stresses in allotetraploid rapeseed. Int J Mol Sci. 2020;21(17):5947.

33. Lescot M, Dehais $P$, Thijs $G$, Marchal $K$, Moreau $Y$, Van de Peer $Y$, et al. PlantCARE, a database of plant cis-acting regulatory elements and a portal to tools for in silico analysis of promoter sequences. Nucleic Acids Res. 2002;30(1):325-7.

34. Ding M, Chen ZJ. Epigenetic perspectives on the evolution and domestication of polyploid plant and crops. Curr Opin Plant Biol. 2018;42:37-48.

35. Song Q, Chen ZJ. Epigenetic and developmental regulation in plant polyploids. Curr Opin Plant Biol. 2015;24:101-9.

36. Yang Z, Gong Q, Qin W, Yang Z, Cheng Y, Lu L, et al. Genome-wide analysis of WOX genes in upland cotton and their expression pattern under different stresses. BMC Plant Biol. 2017;17(1):113.

37. Cheng F, Liang J, Cai C, Cai X, Wu J, Wang X. Genome sequencing supports a multi-vertex model for Brassiceae species. Curr Opin Plant Biol. 2017;36:79-87.

38. Li Y, Meng F, Yin J, Liu H, Si Z, Ni Z, et al. Isolation and comparative expression analysis of six MBD genes in wheat. Biochim Biophys Acta. 2008:1779(2):90-8.
39. Zemach A, Grafi G. Methyl-CpG-binding domain proteins in plants: interpreters of DNA methylation. Trends Plant Sci. 2007;12(2):80-5.

40. Lang Z, Lei M, Wang X, Tang K, Miki D, Zhang H, et al. The methyl-CpGbinding protein MBD7 facilitates active DNA demethylation to limit DNA hyper-methylation and transcriptional gene silencing. Mol Cell. 2015;57(6):971-83.

41. Li K, Ma B, Shen J, Zhao S, Ma X, Wang Z, et al. The evolution of the expansin gene family in Brassica species. Plant Physiol Biochem. 2021;167:630-8.

42. Roulin A, Auer PL, Libault M, Schlueter J, Farmer A, May G, et al. The fate of duplicated genes in a polyploid plant genome. Plant J. 2013;73(1):143-53.

43. Wan Y, Wang Z, Xia J, Shen S, Guan M, Zhu M, et al. Genome-wide analysis of phosphorus transporter genes in Brassica and their roles in heavy metal stress tolerance. Int J Mol Sci. 2020;21(6):2209.

44. Avina-Padilla K, Ramirez-Rafael JA, Herrera-Oropeza GE, Muley VY, Valdivia DI, Diaz-Valenzuela E, et al. Evolutionary perspective and expression analysis of intronless genes highlight the conservation of their regulatory role. Front Genet. 2021;12:654256.

45. Mukherjee D, Saha D, Acharya D, Mukherjee A, Chakraborty S, Ghosh TC. The role of introns in the conservation of the metabolic genes of Arabidopsis thaliana. Genomics. 2018;110(5):310-7.

46. Yang J, Zhou J, Zhou HJ, Wang MM, Liu MM, Ke YZ, et al. Global survey and expressions of the phosphate transporter gene families in Brassica napus and their roles in phosphorus response. Int J Mol Sci. 2020;21(5):1752.

47. Huang Y, He G, Tian W, Li D, Meng L, Wu D, et al. Genome-wide identification of MATE gene family in potato (Solanum tuberosum L.) and expression analysis in heavy metal stress. Front Genet. 2021;12:650500.

48. Li LF, Liu B, Olsen KM, Wendel JF. A re-evaluation of the homoploid hybrid origin of Aegilops tauschii, the donor of the wheat D-subgenome. New Phytol. 2015;208(1):4-8.

49. Bottani S, Zabet NR, Wendel JF, Veitia RA. Gene expression dominance in allopolyploids: hypotheses and models. Trends Plant Sci. 2018;23(5):393-402.

50. Lu K, Wei L, Li X, Wang Y, Wu J, Liu M, et al. Whole-genome resequencing reveals Brassica napus origin and genetic loci involved in its improvement. Nat Commun. 2019;10(1):1154.

51. Scarrow M, Wang Y, Sun G. Molecular regulatory mechanisms underlying the adaptability of polyploid plants. Biol Rev Camb Philos Soc. 2021;96(2):394-407.

52. Bao Y, Hu G, Grover CE, Conover J, Yuan D, Wendel JF. Unraveling cis and trans regulatory evolution during cotton domestication. Nat Commun. 2019;10(1):5399.

53. Gill RA, Scossa F, King GJ, Golicz A, Tong C, Snowdon RJ, et al. On the role of transposable elements in the regulation of gene expression and subgenomic interactions in crop genomes. CRC Crit Rev Plant Sci. 2021:40(2):157-89.

54. Chen X, Tong C, Zhang X, Song A, Hu M, Dong W, et al. A high-quality Brassica napus genome reveals expansion of transposable elements, subgenome evolution and disease resistance. Plant Biotechnol J. 2021;19(3):615-30.

55. Feliner GN, Casacuberta J, Wendel JF. Genomics of evolutionary novelty in hybrids and polyploids. Front Genet. 2020;11:792.

56. Zhang J, Liu Y, Xia EH, Yao QY, Liu XD, Gao LZ. Autotetraploid rice methylome analysis reveals methylation variation of transposable elements and their effects on gene expression. Proc Natl Acad Sci U S A. 2015;112(50):E7022-9.

57. Yu X, Wang P, Li J, Zhao Q, Ji C, Zhu Z, et al. Whole-genome sequence of synthesized allopolyploids in Cucumis reveals insights into the genome evolution of allopolyploidization. Adv Sci (Weinh). 2021;8(9):2004222.

58. Cheng F, Liu S, Wu J, Fang L, Sun S, Liu B, et al. BRAD, the genetics and genomics database for Brassica plants. BMC Plant Biol. 2011;11:136.

59. Lu S, Wang J, Chitsaz F, Derbyshire MK, Geer RC, Gonzales NR, et al. CDD/ SPARCLE: the conserved domain database in 2020. Nucleic Acids Res. 2020;48(D1):D265-8.

60. Mistry J, Chuguransky S, Williams L, Qureshi M, Salazar GA, Sonnhammer ELL, et al. Pfam: the protein families database in 2021. Nucleic Acids Res. 2021:49(D1):D412-9.

61. Blum M, Chang HY, Chuguransky S, Grego T, Kandasaamy S, Mitchell A, et al. The InterPro protein families and domains database: 20 years on. Nucleic Acids Res. 2021;49(D1):D344-54. 
62. Goodstein DM, Shu S, Howson R, Neupane R, Hayes RD, Fazo J, et al. Phytozome: a comparative platform for green plant genomics. Nucleic Acids Res. 2012;40:D1178-86.

63. Artimo P, Jonnalagedda M, Arnold K, Baratin D, Csardi G, de Castro E, et al. ExPASy: SIB bioinformatics resource portal. Nucleic Acids Res. 2012;40:W597-603.

64. Horton P, Park KJ, Obayashi T, Fujita N, Harada H, Adams-Collier CJ, et al. WoLF PSORT: protein localization predictor. Nucleic Acids Res. 2007;35:W585-7.

65. Hu B, Jin J, Guo AY, Zhang H, Luo J, Gao G. GSDS 2.0: an upgraded gene feature visualization server. Bioinformatics. 2015;31 (8):1296-7.

66. Bao W, Kojima KK, Kohany O. Repbase update, a database of repetitive elements in eukaryotic genomes. Mob DNA. 2015;6:11.

67. Qiao X, Li Q, Yin H, Qi K, Li L, Wang R, et al. Gene duplication and evolution in recurring polyploidization-diploidization cycles in plants. Genome Biol. 2019;20(1):38.

68. Wai AH, Waseem M, Khan A, Nath UK, Lee DJ, Kim ST, et al. Genome-wide identification and expression profiling of the $P D /$ gene family reveals their probable involvement in abiotic stress tolerance in tomato (Solanum lycopersicum L.). Genes (Basel). 2020;12(1):23.

69. Librado P, Rozas J. DnaSP v5: a software for comprehensive analysis of DNA polymorphism data. Bioinformatics. 2009;25(11):1451-2.

70. Chen C, Chen H, Zhang Y, Thomas HR, Frank MH, He Y, et al. TBtools: an integrative toolkit developed for interactive analyses of big biological data. Mol Plant. 2020;13(8):1194-202.

71. Kumar S, Stecher G, Li M, Knyaz C, Tamura K. MEGA X: molecular evolutionary genetics analysis across computing platforms. Mol Biol Evol. 2018;35(6):1547-9.

72. Letunic I, Bork P. Interactive tree of life (iTOL) v5: an online too for phylogenetic tree display and annotation. Nucleic Acids Res. 2021:49(W1):W293-6.

73. Bailey TL, Boden M, Buske FA, Frith M, Grant CE, Clementi L, et al. MEME SUITE: tools for motif discovery and searching. Nucleic Acids Res. 2009;37:W202-8.

74. Robert X, Gouet P. Deciphering key features in protein structures with the new ENDscript server. Nucleic Acids Res. 2014;42:W320-4.

75. Li M, Wang R, Wu X, Wang J. Homoeolog expression bias and expression level dominance (ELD) in four tissues of natural allotetraploid Brassica napus. BMC Genomics. 2020;21(1):330.

76. Deng W, Wang Y, Liu Z, Cheng H, Xue Y. Heml: a toolkit for illustrating heatmaps. PLoS One. 2014;9(11):e111988.

\section{Publisher's Note}

Springer Nature remains neutral with regard to jurisdictional claims in published maps and institutional affiliations.

Ready to submit your research? Choose BMC and benefit from:

- fast, convenient online submission

- thorough peer review by experienced researchers in your field

- rapid publication on acceptance

- support for research data, including large and complex data types

- gold Open Access which fosters wider collaboration and increased citations

- maximum visibility for your research: over $100 \mathrm{M}$ website views per year

At BMC, research is always in progress.

Learn more biomedcentral.com/submissions 